\title{
The Evolutionary Development of Human Language
}

\author{
Michael Prost \\ Independent Researcher, Germany \\ Received November 11, 2019; Revised January 7, 2020 ; Accepted February 3, 2020
}

Copyright@2020 by authors, all rights reserved. Authors agree that this article remains permanently open access under the terms of the Creative Commons Attribution License 4.0 International License

\begin{abstract}
One of the most intriguing questions of mankind is the question of how human language evolved. Human language is the primary feature that distinguishes humans from animals. How Homo sapiens acquired language is an open, highly disputed question. So far, science hasn't delivered a satisfactory solution. The most important reason for this failure lies in the fact that no physical evidence for the development of the brain - which plays the central role in the rise of humans - is available. The previous assumption that genetic mutations of the brain were responsible for the evolution of language is not convincing because this would have required two simultaneous genetic mutation of Homo sapiens: a genetic mutation of the brain, and subsequently a mutation of the speaking-apparatus. This seems extremely improbable. We will show here that only the genetic mutation of the speaking-apparatus was necessary. Then two factors came together: in addition to a highly developed brain (which all hominids had and have) an adequate speaking-apparatus was the main factor for the development of language. Upright walking also helped as it enabled them to point with their hands. Some phenotypical mutations also occurred when Homo sapiens started to develop language. One was higher myelinization which improved the signal speed in the human brain compared to other animals. The second one was the development of special areas in the cortex that supported language, understanding and thinking: Wernicke's area and Broca's area. We will also show the connection between human language and human thinking, which is based on the second level of abstraction. This connection proves that the Neanderthals didn't have a sophisticated language. Such a language only developed in Homo sapiens.
\end{abstract}

Keywords Human Language, Evolution, Anatomic Mutations, Neanderthals

\section{Introduction}

One of the most intriguing questions of mankind is the question of how human language evolved. This question came back into the spotlight just a few years ago in the book The Kingdom of Speech written by the well-known and highly acclaimed writer Tom Wolfe (Wolfe 2016)). In the last fifty years the debate was dominated by Noam Chomsky's claim that humans developed a special language acquisition device that lead to a universal grammar among all languages (Chomsky 1955, 1957). Chomsky's view came under pressure after Daniel Everett's elaboration of Pirahã, an indigenous language, which proved that Chomsky's assumption was wrong (Everett 2009). Chomsky himself later retracted his previous view (Chomsky 2006). Now the question is once again completely open. Most scholars assume that human language evolved from pre-linguistic systems among primate ancestors (Pinker 1994, Ulbaek 1998). As the distinctive feature some prefer genetic changes (Päabo 2014), some assume social communications (Tomasello 2013), while some rely on anatomic mutations (Liebermann 1975, 2006). A complete overview of the different positions and aspects can be found in the Oxford Handbook of Language Evolution (Tallermann 2012). So far, no position is entirely persuasive. One scholar who covers at least one aspect is Yuval Noah Harari (Harari 2019). Harari realizes the importance of the development of the human language for the development of mankind. He identifies the cognitive revolution which led to what he calls "fictive thinking". That is what we will denote as "second level of abstraction" and will explain in detail, below. But also Harari admits that nobody knows how this happened. First, we will show here that a combination of different factors - namely a developed brain, mutations in the speaking-apparatus and the ability to point - ultimately led to the development of today's human language. We will illustrate the development by first explaining animals' signal language. Based on these explanations we will describe the evolutionary development of the human language. Finally, we will prove our explanation by looking at the Neanderthals.

\section{Animals' Signal Language}

When we want to understand the development of the 
human language we first have to understand the development of animals' signal languages. When we want to understand the development of animals' signal languages we have to take a deeper look into the specifics of the brain of the families humans belong to, namely mammals, especially primates. As it is known, we share many genes with other primates; with chimpanzees we have more that $98 \%$ genes in common. Generally, all mammals have a brain. The brain is the organ that controls all body functions and all actions. The brain is constituted of cells called neurons and glia cells. The glia cells are responsible for the nourishment of the neurons and isolate the neurons from one another. For the functioning of the brain only the neurons play a role. The brain functions via connections between the neurons and signal transmissions along these connections. The brain works in three modes: unconsciousness, subconsciousness and consciousness. Unconsciousness is a mode that only uses fixed neural connections, for example for the control of heartbeat or of digestion. The vast majority of all brain functions are performed unconsciously. Only in one part of the brain is the situation different, namely in the cerebrum, and there only in a special part called cortex. The cortex exhibits a property called neuroplasticity. Neuroplasticity is the ability of the cortex to create new neural connections based on internal or external sensations. The cortex can work in two modes, namely subconsciously and consciously. First, a sensation creates a neural connection, a neural pattern, that is characteristic for this sensation. For example, images create specific neural patterns in the visual cortex; sounds create specific neural patterns in the auditory cortex. Based on sensations, more neural connections can be activated that ultimately send signals to muscles, thus initiating actions. Subconsciously initiated actions are basically reflexes. Consciously activated actions can be considered as acts of thinking. As long as these actions are based on sensations this kind of thinking must be called sensory thinking. That means all mammals, including primates, are capable of sensory thinking. If we consider the hunting of cats, we immediately realize that this behavior is logical and rational. Now it has to be emphasized that this behavior is not innate but must be learned. Consequently, consciousness is also the basis for learning.

Also consequently, consciousness is then the prerequisite for the development of signal languages. What is a signal language? As the name itself suggests, the capability of signal languages is limited to transmitting signals. A good example for animals' sensory thinking and their signal language can be found among vervet monkeys. Vervet monkeys live in trees and use different signals when threatened. They can be threatened by a predator on the ground, a snake in the tree or a raptor in the air. These various types of threats require different reactions; consequently, they are initiated by different signals. If threatened by a leopard on the ground, an appropriate signal motivates all monkeys to climb the tree to some small branches that would not hold the weight of the leopard. If threatened by a snake that lives in the tree an appropriate signal motivates them to leave the tree. And if threatened by a raptor, an appropriate signal motivates them to hide under the leaves in the tree. This example shows that the behavior of vervet monkeys is intelligent, but it also outlines two features which are characteristic for all signal languages. All signals are connected to imaginations and all signals represent a certain kind of abstraction. The signal for snake is connected to the image of a snake. When a snake is seen a connection will be made to the signal for snake. Now we have to be aware that all snakes are different. But on the other hand, all snakes have something in common which differentiates them from other creatures. One could say that it is the sighting of one member of the set of snakes that initiates the alarm signal. This is an act of abstraction. Summarizing, we can say that signal language represent an act of abstraction, but all signals are connected to imaginations.

Now we can ask what is necessary for the development of a signal language. There are two prerequisites: first, a sophisticated brain that is capable of distinguishing the different sounds and to align them with characteristic imaginations and appropriate actions. But second, there must also exist the anatomic possibility of the speaking-apparatus (=all parts of the head that are used to create sounds) to produce characteristic sounds. We need to look at this process in detail. The sight of the leopard creates a visual neural pattern that represents the leopard in the visual cortex. Once a monkey has sighted a leopard he must then create an appropriate signal. For this, he needs a neural connection from the visual neural pattern for leopard to another neural connection, another neural pattern, that sends a signal to the speaking-apparatus which now produces the characteristic sounds. Because this second neural pattern initiates a muscle movement it must be located in the motor cortex. We can call it a neural speak-pattern. Now, the other monkeys hear the signal and the signal creates another different neural pattern, this time in the auditory cortex. These neural patterns are called auditory neural patterns. The other monkeys then connect the perceived auditory neural patterns with the visual neural pattern that represents the image of a leopard. They now know what the signal means. By learning, they have built more neural connections that now motivate them to climb the tree to some small branches. To summarize: An external image creates a visual neural pattern in one monkey. This visual neural pattern is then connected to a neural speak-pattern; consequently, the monkey creates a sound, a signal. This signal creates auditory neural patterns in the other monkeys. There these auditory neural patterns will be connected with the visual neural pattern of the leopard and will subsequently connect to more neural patterns in the motor cortex which finally motivate the monkeys to move to protection in the tree. From these explanations it should also be clear that the signal-language has to be learned and is not an innate feature.

We have described here how a signal language works. 
Now we have to describe how the sigal language evolved. The evolutionary development happened over an extended period of time. At the beginning, one member of the species created a signal for the first time. At this time, the other members of the group did not know what the signal was supposed to mean. But after some time, they understood and started to create the same signal in a similar situation. After the successful introduction of one signal, more signals were invented. Finally, every member of the group understood the different signals and could also produce them. This knowledge had to be passed on from one generation to the other and finally spread out through the entire species.

\section{The Development of Human Language}

First, we must assume that all predecessors of the modern human, all Hominini, also developed a signal language. But as we indicated above, all attempts by scientists to explain how Homo sapiens developed his sophisticated declarative language have failed so far. We humans have a huge amount of knowledge, expressed in human language, but we don't know why and how we developed the language. The reason for the failure is that scientists have been asking the wrong questions. They always asked what developments of the brain lead to the development of language. But the answer is surprising: there were no initial significant mutations of the brain that led to the development of language. The Homo neanderthalensis, who evolved earlier from Homo erectus also a predecessor of Homo sapiens - developed a brain that was even bigger than that of Homo sapiens, but as we will show below he didn't develop a sophisticated declarative language like Homo sapiens did. So what was then the reason that Homo sapiens developed the human language?

As we mentioned in connection with signal languages, there is a second requirement for the development of a language besides an appropriate brain: an appropriate speaking-apparatus. Consequently, the correct answer to the question above is: through mutations Homo sapiens developed an adequate speaking-apparatus to produce enough distinguishable memorizable sounds. Only because of these mutations of the speaking-apparatus was Homo sapiens able to develop the sophisticated human language. We can illustrate the principle that an adequate speaking-apparatus is needed if we assume that we would hypothetically only be able to create the five vowels. Of course, five vowels can create an infinite number of combinations. (Actually, as few as two sounds can already do this: that is binary language which is used in all computers.) That means one would be able to create an infinite number of different terms, which is sufficient to create any sophisticated language. But the caveat is that the human brain or any other brain would not be able to remember all these combinations and thus would realistically reduce the possibility of sophisticated language, even if it were theoretically possible. It would certainly be feasible for the brain to associate different meanings with the combinations 'AUI', 'OUI', 'EIA' or even 'AIEU'. But if the combinations 'AUIEEAUOEI' or 'OIUAEEOIU' are formed, the brain will not be able to remember the possible meaning of those combinations. The development of any language would be limited to the degree we see in animals' signal-language. But Homo sapiens has experienced major mutations and changes of the speaking-apparatus compared not only to chimpanzees, but also compared to earlier species of Homo. The major change is the lowering of the larynx, accompanied by more changes such as the enlargement of the pharynx and a repositioning of the hyoid bone (Liebermann 1975, Liebermann 2006, Ghazanfar 2008). These evolutionary changes gave Homo sapiens a previously unknown ability to create different sounds. Consequently, the mutations of the speaking-apparatus were the decisive factor that led to the development of the human language and not, as many scientists have long assumed, mutations of the brain.

And another anatomic specialty benefited the development of human language: the fact that humans are bipeds. The creation of sounds does not guarantee that the other members of the group know what the new sound is supposed to denote. This is where pointing comes into play. Humans had free arms, hands and fingers and could use these extremities to point to objects which should be associated with the new sounds. They could point to a mango and say "mango". They could point to a banana and say "banana". They would have even been able to make a circular movement of their hands over a pile of mangos and bananas and say "fruit", thereby starting to facilitate the second level of abstraction. (We will discuss the second level of abstraction soon.) To illustrate the importance of pointing we will use the example of you walking along the beach with another person who speaks a foreign language that you don't know, e.g. German. Suddenly the person says "Baum". What does she mean? Does she mean the beach, the sand, the water, the sea, the sky or something else? If she now points at a tree, you immediately know that "Baum" means "tree". But as we will see later when we discuss the Neanderthals it should be emphasized that the ability to point was just a helpful feature, the ability of the speaking-apparatus remains the decisive necessary factor.

Generally, we can assume that human language started as signal language, too. We can also assume that human language started to produce more signals than signal languages of other animals. But there was one decisive point when human language began to be completely different and that was the invention of the second level of abstraction. As we have seen, the first level of abstraction already occurs in animals' signal language. In our example of the vervet monkeys, the first level of abstraction would be the signals for raptor, snake or leopard. In human language the first level of abstraction could have been to associate signals with certain fruits, e.g. banana, pineapple or mango. But at some 
point, someone figured out that these different kinds of fruits had something in common. Consequently, she created a new signal for the class of these objects, namely the term "fruit". This is the second level of abstraction because the term "fruit" is no longer connected to an imagination. The meaning of the term "fruit" can only be understood by definition which requires the use of other terms. The importance of the second level of abstraction is that it now became possible to invent time as part of language. The first level of abstraction is always connected with "present". The second level of abstraction now made it possible to add "past" and "future" to language. This again made it possible to include "recursion" in language, and this again made it possible to develop an understanding of "causality". The mastery of the concept of "causality" is the foundation of human civilization and can even be assumed to be the foundation of any possible intelligent civilization in the universe. Causality is the basic concept behind the development of the universe. Consequently, it is the invention of the second level of abstraction which distinguishes human language from any other animals' language and gives human thinking a completely different advanced capability. We have to emphasize here that verbal thinking is silently speaking to ourselves. We experience this when we think: we speak silently to ourselves. This new, human kind of thinking is now based on language and on the second level of abstraction and can be called "verbal thinking". Consequently, development of a declarative language and advanced intellectuality are closely connected.

Now, as always in evolution, a long process of development started. Mutations of the speaking-apparatus gave Homo sapiens the possibility to develop more words and gave her an evolutionary advantage. For hunting it was beneficiary to have information about possible prey. One impala at the distance was not worth going after, because the potential quantity of meat was too small. A small group of impala at some distance was worth going after, because there was a chance to catch several animals. A big group of impala at some distance was not worth going after, because the chance to be spotted by one animal of the group was too big. Another advantage occurred on a social level. Without language, it is only possible to make conclusions about a person's mood by her behavior. But this can be ambiguous. Is a quiet person sad or ill? If the person can speak she can say how she feels and can be treated in a proper way.

Now we want to understand how everything is realized in the brain. We have already outlined the principles in our example above. We have already learned that three different kinds of neural patterns - one primary neural pattern and two neural language-patterns - have to be connected. To extend the number of language-patterns to the amount we humans have today another parallel evolutionary development was necessary. In order to connect three neural patterns simultaneously a higher neural transmission rate was needed. This became possible through a higher myelinization. Myelin is a fatty white substance that surrounds the axon of neurons, forming an electrically insulating layer. The myelinization in the human brain is much higher than in any other animals. That leads to much higher neural transmission speed in human brains. The higher myelinization need not be due to genetic mutations; it could even be caused by phenotypical changes. Consequently, the entire process of development of human language was iterative. Some mutations of the speaking-apparatus occurred, enabling the bearer of these mutations to invent and produce more words. Faster neural transmission was necessary to process these additional words. Thus, the members of Homo sapiens who underwent the mutations of the cortex with higher myelinization had an advantage. Now some of these people experienced further mutations of the speaking-apparatus, again improving the ability to produce more sounds. Then again, people with still higher myelinization were better equipped to process the bigger vocabulary. And as outlined above, with more language the ability of verbal thinking also improved. But we have to emphasize here two things. As mentioned, the mutation of the brain to a higher myelinization need not have been genetic, it could also have been just a mutation of the phenotype. These mutations can occur much easier than genetic mutations. Second, the mutation of the brain to higher myelinization was the result of the mutation of the speaking-apparatus, not vice versa. Without the mutation of the speaking-apparatus a higher myelinization would have been useless.

Generally speaking, we can emphasize once again that the mutations of the speaking-apparatus were the decisive factor in the development of the human language. Let's assume there were only a few critical mutations of the brain required for the brain to develop a declarative language. But these abilities of the brain would have been useless if they were not accompanied by a speaking-apparatus that was able to create enough distinguishable and memorizable sounds. Consequently, the previous assumption of most scholars that genetic mutations of the brain led to the development of language and thinking is almost certainly wrong. This assumption would have required first a genetic mutation of the brain which would have led to the formation of language supporting structures like the Broca's area and the Wernicke's area. But this would not have been sufficient. Simultaneously genetic mutations of the speaking apparatus would also have been required. It is known that the speaking apparatus of other primates (including all other hominini) is / was not able to produce the variety of sounds that Homo sapiens can. (We will discuss the Neanderthals below.) A smart geneticist could certainly calculate the probability that both genetic mutations could happen simultaneously. The author assumes that this probability is nearly zero. Vice versa, we have a completely different story. After the genetic mutations of the speaking-apparatus the brain could develop smoothly. It was easy for neuroplasticity to develop a structure like the Wernicke's area which is located in the parietal lobe close to the auditory cortex and which plays a critical role in understanding. It was also easy for 
neuroplasticity to develop a structure like the Broca's area which is located in the frontal lobe close to the motor cortex and which plays a critical role in reasoning and formulating language. Both, the development of the Wernicke's area as well as the development of the Broca's area didn't need any genetic mutations.

Now the objection might arise that the given explanation is somewhat speculative, and that more experimental evidence is required. The caveat is: there is no evidence and there can never be any evidence. The reason, of course, is that no remains of human brains (or of brains from other animals) on the appropriate time scale are available. The only remains of hominini are bones and tissue. Consequently, all theories about how brains developed are speculative. That is the reason why all theories about the evolution of the human brain are speculative. That again is why plausibility is a criterion for judging these theories. As we have just explained, two kinds of mutations occur in the evolutionary process. One kind is genetic; the other kind is phenotypical. Genetic mutations occur purely accidental. As pointed out, this means that if the development of the Wernicke's area and the development of the Broca's area were genetic another genetic mutation, the mutation of the speaking-apparatus, would have been required. That is completely improbable. On the other hand, the reverse namely first the genetic mutation of the speaking-apparatus and subsequent phenotypical developments of the brain and the Wernicke's area and the Broca's area - is very reasonable. Because we unfortunately have to accept that no physical evidence will ever be available for any theory about the development of the human language and that we will have to rely on plausibility. Consequently, it is also plausible to assume that the generally missing physical evidence is the reason that so far no satisfying explanation for the evolution of the human language has been proposed.

\section{The Neanderthals}

As we have already mentioned above, we can strongly assume that the Neanderthals didn't develop a sophisticated language. (Harari makes the same assumption.) Why this assumption? Because the Neanderthals went extinct. Why do we have to assume that the extinction of the Neanderthals proves that they did not develop a sophisticated language? Obviously the Neanderthals had a limited intellect. Homo sapiens evolved 150,000 to 200,000 years ago from Homo erectus in Africa. At the beginning Homo sapiens used the same tools that Homo erectus had developed before. Then Homo sapiens started to develop new tools, using his emerging capabilities of language and thinking. As we know it took Homo sapiens 150,000 to 200,000 years to further develop telephones, television, refrigerators, cars, airplanes and even rockets and satellites. How was the situation with the Neanderthals? During the 400,000 years between their first appearance up and their extinction the Neanderthals developed: nothing! The Neanderthals were hunters as all Hominini. As hunters they consumed meat. Moreover, the overwhelming part of their diet was meat to which they only added tiny amounts of vegetarian components. This diet contributed to the development of a big brain. But though the brain of the Neanderthals was bigger than the human brain it was obviously not as efficient. It is known that the Neanderthals used the same kind of spears for hunting at the time of their extinction that were already used 400,000 years ago. This obviously worked quite well for a long time. But then change and further development of tools would have been required. The characteristics of the spears of the Neanderthals were that they were only usable for direct impact. The Neanderthals approached their prey until they were close enough to stab it with the spears. Because their prey were large animals such as mammoth, giant elk (Megaloceros) and wooly rhinoceros these animals tolerated a close approach by the hunters. But they also did fight when attacked. Many skeletons of Neanderthals show that they had broken bones: a consequence of their hunting method. Now something happened at the end of the last ice age that proved to be fatal for Neanderthals. The large animals went extinct or became less abundant. The Neanderthals could no longer find sufficient prey. Now they would have been forced to develop their spears into tools that could also be used for throwing. Then they could have included smaller animals into their diet. The smaller animals could not be hunted with the traditional spears because they would not allow hunters to come close enough. Maybe the Neanderthals now included more vegetarian components into the diet. But without enough vitamin B12 that was only available in meat the infants could not grow up anymore. In the authors opinion, that was the reason they went extinct. It can be assumed that Homo sapiens integrated some remaining members of the Neanderthals. This would explain why the modern human still carries some Neanderthal genes (This is of course only true for European and Asian humans where Homo sapiens and Neanderthals lived together at the same time.) The conclusion is that the Neanderthal had a big brain which might have been sufficient to develop a sophisticated language, but they didn't because they didn't have the necessary mutations of their speaking-apparatus. Consequently, they stayed limited in their intellectual capacity.

Now we can still ask why the Homo sapiens had the mutations of the speaking apparatus but the Neanderthals did not. That is, of course, because the Neanderthals evolved from Homo erectus already almost 200000 years before Homo sapiens. They migrated to Europe and Asia and thus were genetically completely separated from Homo sapiens. Homo sapiens developed from the African line of Homo erectus about 200,000 to 150,000 years ago and we can be pretty sure that the origin of the development of human language lies in today's Namibia / Botswana. There and at that time the aforementioned mutations of the speaking-apparatus occurred and Homo sapiens began to 
develop an advanced declarative language. We know this because every language has some elements called "phonemes", which are small units of any language that give terms different meanings. For example, the terms "kiss" and "kill" have different meanings because the phoneme "ss" is replaced by the phoneme "ll". Every language has a certain numbers of phonemes. Studies (Atkinson 2012) have found out that the number of phonemes decreased with the propagation of Homo sapiens. The language with the most phonemes is !Xóõ, a language spoken in Namibia / Botswana. That is a language which still contains clicks. (There is still an amazing connection to humans everywhere else in the world. It can be assumed that clicks were originally used for communications during hunting. Clicks don't alert animals that much. And still today, everywhere in the world clicks are used for communication between hunters!) The language with the least phonemes is Hawaiian, spoken on Hawaii. Actually, Hawaii was the last piece of earth where humans settled. If one now follows the path of human propagation one sees that the number of phonemes decreases the farther away Homo sapiens settled from her origin in Africa, namely Namibia / Botswana.

It took Homo sapiens about 140,000 to 190,000 years until human language and the ability to think was so far developed that we had the intellectual capacity to start agriculture and livestock farming and to settle down. (That happened about 10000 B.C.) With settlements, agriculture and livestock farming Homo sapiens was able to assign different tasks to different members of the group. Before that, all men went hunting and all women were in charge of preparing food and raising the children. With the dedication of different tasks to different members of the group a new level in the development of human language and of human knowledge began. From that point on, it took humankind about 12,000 years to bring people to the moon. In conclusion we can say that a sufficiently developed brain is necessary for the development of a sophisticated language. But that is not the decisive factor because we can assume that Homo erectus most likely had such a brain and even chimpanzees might have it. The decisive factor is that the anatomy of the speaking apparatus is able to create a large variety of different sounds. After this critical mutation happened to Homo sapiens she could start to develop an advanced language and advanced thinking.

\section{Summary}

The question how Homo sapiens evolved and developed language and abstract thought is one of the most intriguing unresolved riddles of science. This is actually somewhat embarrassing because humans should know what led to their special role on the planet, and maybe even in the Galaxy or in the universe. The most important reason for this failure lies in the fact that no physical evidence for the development of the brain - which plays the central role in the rise of humans
- is available. This leaves speculation as the only tool to try to explain human development. But all recent theories have exhibited one decisive failure: they all concentrate solely on the potential evolutionary development of the brain as the source of the development of human language and human thinking. This article takes a different approach and brings a complete new perspective into play. It takes into consideration all the developments that were necessary for the development of human language, leading to the conclusion that the evolutionary development of the speaking-apparatus in Homo sapiens was the decisive factor and that the development of the brain happened subsequently, requiring only phenotypical changes as opposed to genetic mutations. Consequently, the development of advanced human language (and advanced human thinking) only occurred in Homo sapiens. The development of human language led to the decisive juncture where advanced thinking - verbal thinking - started, namely to the second level of abstraction. The further development of the second level of abstraction led to the concept of time as a part of language, to the recognition of causation and finally to the development of human culture and human technology.

Summarizing it can be said that this article presents for the first time a conclusive explanation of the evolutionary development of human language, and subsequently of human thinking.

\section{Acknowledgements}

I would like to thank Prof. Dr. Vincent Marchesi, Anthony N. Brady Prof. of Pathology and Professor of Cell Biology, Director Boyer Center for Molecular Medicine, Yale University, for pointing out to me the importance of the high human myelinization.

\section{REFERENCES}

[1] Atkinson, Q. Phonemic diversity supports a serial founder effect model of language expansion from Africa. Science, Vol. 332, No. 6027, pp. 346 - 349 (2012)

[2] Chomsky, N., The Logical Structure of Linguistic Theory. (University of Chicago Press, Chicago 1955)

[3] Chomsky, N. Syntactic Structures. (De Gruyter Mouton, Boston 1957)

[4] Chomsky, N. Language and Mind. (Cambridge University Press, Cambridge 2006)

[5] Everett, D. Don't Sleep There are Snakes. (Profile Books, London 2009)

[6] Ghazanfar, A., Rendell, D. (2008) Evolution of Human Vocal Production, Current Biology, Volume 18 (11), R457-R460, (2008) 
[7] Harari, Y.N. Sapiens - A Brief History of Humankind. (Harper Perennial, New York, 2019)

[8] Lieberman, P. On the Origins of Language. (Macmillan, New York 1975)

[9] Lieberman, P. Towards an Evolutionary Biology of Language. (Harvard University Press, Cambridge 2006)

[10] Päabo, S. Neanderthal Man: In Search of Lost Genomes. (Basic Books, New York 2014)

[11] Pinker, S. The language instinct. (W. Morrow and Co, New York 1994)

[12] Tallerman, M., Gibson, K. The Oxford Handbook of Language Evolution. (Oxford University Press, New York 2012)

[13] Tomasello, M. Constructing a Language: A Usage-Based Theory of Language Acquisition. (Harvard University Press., Cambridge 2013)

[14] Ulbæk, I., Hurford, J.R., Studdert-Kennedy, M., Knight, C. eds., The origin of language and cognition. in Approaches to the evolution of language: social and cognitive base. (Cambridge University Press, New York 1998)

[15] Wolfe, T. The Kingdom of Speech. (Little, Brown and Company Boston 2016) 